\title{
Simvastatin Attenuates Hippocampal MMP-9 Expression in the Streptozotocin-Induced Cognitive Impairment
}

\author{
Soheila Adeli ${ }^{1,2}$, Maryam Zahmatkesh $^{1,2,3^{*}}$ and Mitra Ansari Dezfouli ${ }^{1}$ \\ ${ }^{1}$ Department of Neuroscience and Addiction Studies, School of Advanced Technologies in Medicine, \\ Tehran University of Medical Sciences, Tehran, Iran; ${ }^{2}$ Electrophysiology Research Center, Neuroscience \\ Institute, Tehran, Iran, Tehran University of Medical Sciences, Tehran, Iran; ${ }^{3}$ Research Center for \\ Cognitive and Behavioral Sciences, Tehran University of Medical Sciences, Tehran, Iran
}

Received 24 June 2018; revised 31 July 2018; accepted 6 August 2018

\begin{abstract}
Background: Matrix metalloproteinase-9 (MMP-9) expression has been implicated in molecular mechanisms of neurodegenerative disorders, and its abnormal level has been reported in Alzheimer's disease (AD). Some protective mechanisms of statins against neurodegeneration might be mediated by the inhibition of MMP-9 expression. Here, we investigated the effect of simvastatin on the hippocampal MMP-9 expression in the context of AD. Methods: We examined the influence of three-week simvastatin $(5 \mathrm{mg} / \mathrm{kg})$ administration on hippocampal MMP-9 expression in a rat model of cognitive decline induced by streptozotocin (STZ). Spatial long-term memory and MMP-9 expression were assessed by Morris water maze (MWM) test and quantitative polymerase chain reaction, respectively. Results: The results showed a decline in the learning and memory in STZ group when compared with the control group. The MMP-9 up-regulated $(1.41 \pm 0.2 \mathrm{vs.} 0.980 \pm 0.02, p<0.05)$, and cresyl violet staining showed hippocampal cell damage in STZ group compared with the control group. Simvastatin prevented the up-regulation of MMP-9 $(1.05 \pm 0.05$ vs. $1.41 \pm 0.2, p<0.05)$, improved spatial memory impairment and attenuated hippocampal cell damage. Furthermore, we found a negative correlation $(r=0.77)$ between MMP-9 expression and cognitive function. Conclusion: Our findings suggest that the neuroprotective influence of simvastatin in battle to cognitive impairment is mediated in part by the modulation of MMP-9 expression. The reduction of MMP-9 expression in simvastatin-treated animals is in correlation with the improvement of cognitive functions. Understanding the protective mechanism of simvastatin will shed light on more efficient therapeutic modalities in AD. DOI: 10.29252/ibj.23.4.262
\end{abstract}

Keywords: Alzheimer's disease, Matrix metalloproteinase-9, Simvastatin

Corresponding Author: Maryam Zahmatkesh

Department of Neuroscience and Addiction Studies, School of Advanced Technologies in Medicine, Tehran University of Medical Sciences, Tehran, Iran; Tel.: (+98-21) 43052155; Fax: (+98-21) 88991117; E-mail: zahmatkm@tums.ac.ir

\section{INTRODUCTION}

$\mathrm{M}$ atrix metalloproteinases-9 (MMP-9), also known as gelatinase $\mathrm{B}$, is a zinc-dependent endoprotease. Its expression has been implicated in molecular mechanisms of many pathological processes affecting the central nervous system $^{[1]}$. Considerable researches have been conducted on the role of MMP-9 in neurodegenerative disease $^{[1,2]}$. In Alzheimer's disease (AD), the higher levels of MMP-9 have been reported in the plasma ${ }^{[3,4]}$ and hippocampus ${ }^{[5,6]}$. It has been shown that MMP-9 activity in the frontal and parietal cortex is greater in both $\mathrm{AD}$ and mild cognitive impairment compared to healthy subjects ${ }^{[7]}$. Increased hippocampal MMP-9 expression is involved in the development of cognitive impairment induced by beta-amyloid ${ }^{[8]}$. It has been demonstrated that laminin degradation by MMP-9 induces neuronal damage ${ }^{[9]}$.

Clinical administration of statins is associated with the reduction of the AD incidence later in life ${ }^{[10]}$. Statins are HMG-CoA (3-hydroxy-3-methylglutaryl 
coenzyme A) reductase inhibitors commonly used for managing hypercholesterolemia. Simvastatin is a hydrophilic statin that can cross the blood brain barrier and has neuroprotective properties ${ }^{[11]}$. It exerts the strong antioxidant effect in the nervous system ${ }^{[12]}$, attenuates glial activation ${ }^{[13]}$ and reduces the expression of neuro-inflammatory mediators ${ }^{[14,15]}$. Moreover, simvastatin has been shown to effectively protect blood brain barrier integrity ${ }^{[16,17]}$ and decrease the mRNA levels of MMP-9 in in vitro studies ${ }^{[18,19]}$. Various studies have focused on MMP-9 gene expression in neurodegenerative disorders ${ }^{[8,20]}$. It has been reported that some protective mechanisms of simvastatin may be mediated by the inhibition of MMP-9 gene expression ${ }^{[21]}$. There is no specific study centering the neuroprotective effect of simvastatin on memory performance in line with MMP-9 gene expression in the context of AD. In the present study, we evaluated the effect of oral simvastatin on hippocampal MMP-9 expression in a rat model of cognitive impairment induced by streptozotocin (STZ) infusion into the cerebral ventricles (ICV).

The ICV-STZ injection is a rat model of sporadic $\mathrm{AD}$ for preclinical testing of pharmacological therapies against $\mathrm{AD}^{[22,23]}$. The ICV-STZ injection decreases the cerebral glucose uptake, desensitizes brain insulin receptors, decreases the PI3K-AKT signaling activity and increases the activity of glycogen synthase kinase 3 beta $^{[23,24]}$. These changes ultimately promote tau hyper-phosphorylation. Moreover, glucose hypometabolism initiates the process that ultimately results in $A \beta$ aggregation $^{[24]}$ and induces memory impairment similar to sporadic $A \beta$ pathology ${ }^{[25,26]}$. In the current study, we examined the effect of simvastatin administration on hippocampal MMP-9 expression in an animal model of cognitive impairment induced by intracerebroventricular (ICV) streptozotocin (STZ) administration.

\section{MATERIALS AND METHODS}

\section{Animals}

Male Albino Wistar rats, weighing 280-290 g, were used in all experimental procedures. Rats were kept under a controlled condition at $22 \pm 2{ }^{\circ} \mathrm{C}$ and had free access to rat chow and water in their cages in a $12: 12$ hour light/dark cycle beginning with lights on at 7:00 am. All experiments were done in accordance with the guideline for the use of laboratory animals of the National Institutes of Health and approved by the Research and Ethics Committee of Tehran University of Medical Sciences, Tehran, Iran. All behavioral tests were performed between 9:00 am and 12:00 am.

\section{Brain surgery}

For stereotaxic surgery, anesthesia was induced with intraperitoneal injection of ketamine and xylazine (60 $\mathrm{mg} / \mathrm{kg}$ and $15 \mathrm{mg} / \mathrm{kg}$, respectively; Alfasan, Woerden, Holland), and animals were positioned in the stereotaxic device (Stoelting Inc., USA). Stereotaxic coordinates for lateral ventricles were chosen $(1.5 \mathrm{~mm}$ lateral to sagittal suture; $-0.8 \mathrm{~mm}$ to Bergman; and 4 mm under the brain surface) according to the atlas of rat brain (Paxinos and Watson 2007). Guide cannulas were implanted bilaterally $1 \mathrm{~mm}$ above the lateral ventricles. The guide cannula was fixed with dental cement. During surgery, body temperature was kept at $36.5 \pm 0.5{ }^{\circ} \mathrm{C}$ using a heating pad. After surgery, $50 \mathrm{mg} / \mathrm{kg}$ ampicillin was administered intramuscularly.

\section{Microinjection procedure}

One week after surgery, general activity was assessed with the open field test. The exclusion parameters in the experiments were lack of normal locomotor activity, weight gain, and general health condition. STZ (1.5 mg/5 $\mu \mathrm{L} / \mathrm{side}$; Alexis, Lausen, Switzerland) or saline ( $5 \mu \mathrm{L} /$ side $)$ were infused on days 1 and 3 using a needle $1 \mathrm{~mm}$ longer than the guide cannula. The needle was attached to a polyethylene tube connecting to the 5- $\mu$ l Hamilton microsyringe. Microinjections were performed slowly $(0.4 \mu \mathrm{L} / \mathrm{min})$, and the injection needle was left in place for a few additional minutes to avoid the backflow of injected materials. During the microinjections, the animals were free in their cage.

\section{Oral administration of simvastatin}

Simvastatin (ab120505; Cambridge, UK) was dissolved in dimethyl sulfoxide (DMSO) 1\%, and administered $5 \mathrm{mg} / \mathrm{kg}$ body weight through an oral gavage for three weeks after the first injection of STZ.

\section{Experimental groups}

Rats were randomly assigned into four groups (eight rats per experimental group) as follows: (1) Saline + DMSO or vehicle group that received bilateral ICV infusion of saline (STZ vehicle) on days one and three and oral gavage of DMSO 1\% (simvastatin vehicle) daily for three weeks; (2) STZ + DMSO group that received bilateral ICV infusion of STZ on days one and three and oral gavage of DMSO daily for three weeks. (3) STZ + Sim group that received bilateral ICV infusion of STZ and oral gavage of simvastatin daily for three weeks; (4) Saline + Sim group that received bilateral ICV infusion of saline on days one and three and oral gavage of simvastatin daily for three weeks. 


\section{Spontaneous motor activity}

Locomotor and exploration activity were evaluated with open field test as previously described ${ }^{[27]}$. The apparatus was made of a field measuring $80 \times 80 \mathrm{~cm}$ surrounded by $40-\mathrm{cm}$-high walls. Animals were placed in the center of the open field device and allowed to move freely for five minutes. The incidence rate of line crosses and the number of rearing were measured to determine locomotor activity. The field was cleaned with $70 \%$ ethanol at the end of each session.

\section{Learning and memory assessment}

All 32 rats were subjected to the behavioral tests. We evaluated spatial long-term memory using MWM test $^{[28]}$. A black water tank $(140 \mathrm{~cm}$ in diameter, $60 \mathrm{~cm}$ high, and depth of $30 \mathrm{~cm})$ was filled with water $(28 \pm 1$ $\left.{ }^{\circ} \mathrm{C}\right)$. The water pool was placed in a silent room with several spatial cues. The water pool was divided into four equal parts. In the place navigation stages, the transparent escape platform $(10 \mathrm{~cm}$ in diameter) was positioned at the midpoint of one quadrant. The rat could climb on the platform to escape from swimming. Each rat received four trials per day on days 17, 18, 19, and 20 after STZ injection. In each trial, the starting quadrant was selected randomly, and a maximum time of $60 \mathrm{~s}$ was given to rats to find the platform, and then they were allowed to stay on it for five s. On the first day, the rats were subjected to trials with visible platform. On the next three days, the platform was submerged below the water surface. Latency to escape from the water maze and the swimming distance to find the hidden platform were registered for each trial. Twenty-four hours after the last trial session, the platform was removed, and the probe test was done. The time spent and the distance traveled by the rat in the goal quadrant (where the platform was located during hidden platform training) were calculated. All data were recorded using video-tracking software (Radiab1 v2, Iran), which monitored the movements of rats above the center of the pool.

\section{RNA extraction, cDNA synthesis, and quantitative} polymerase chain reaction ( $q$ PCR)

At the end of the experiment, the brains of all animals were removed. The hippocampus tissues of four animals in each group were used for RNA extraction and PCR analysis. Hippocampal total RNA was extracted using Trizol reagent (Zist Abzar Pajoohan Co., Iran). Purification of total RNA was evaluated using the Nanodrop 1000 Spectrophotometer (Thermofisher Scientific, USA). RNA quality was estimated by depicting $18 \mathrm{~S}$ and $28 \mathrm{~S}$ ribosomal RNA bands through electrophoresis. Then $500 \mathrm{ng}$ of total RNA was reverse transcribed into cDNA using the
PrimeScript RT kit (Thermofisher scientific) as stated by manufacturer's protocol. Primers, RNA template, and nuclease free $\mathrm{H}_{2} \mathrm{O}$ mixture were kept warm at 65 ${ }^{\circ} \mathrm{C}$ for about $5 \mathrm{~min}$. Subsequently, the solution was mixed with $2 \mu \mathrm{L}$ of $5 \times$ PrimeScript buffer solution and incubated at $37{ }^{\circ} \mathrm{C}$ for $15 \mathrm{~min}$ and $85{ }^{\circ} \mathrm{C}$ for $5 \mathrm{~s}$ and preserved at $-20^{\circ} \mathrm{C}$ until further use. The resulting cDNA was used for quantitative measurement of gene expression levels of MMP-9 and GAPDH (glyceraldehyde 3-phosphate dehydrogenase) using the SYBR Premix Ex Taq II kit (Takara, Japan). The cycling conditions were $95{ }^{\circ} \mathrm{C}$ for $3 \mathrm{~min}, 35$ cycles at $95^{\circ} \mathrm{C}$ for $10 \mathrm{~s}, 59^{\circ} \mathrm{C}$ for $10 \mathrm{~s}$, and $72^{\circ} \mathrm{C}$ for $35 \mathrm{~s}$. Realtime PCR was done in a real-time qPCR cycler instrument (Rotor-Gene Q, Qiagen), and the threshold cycle $(\mathrm{Ct})$ was applied to quantify the mRNA level. Relative gene expressions were analyzed by $2^{-\Delta \Delta C t}$ method, normalized to GAPDH, a housekeeping gene, and relative to the control group ${ }^{[29]}$. The primers used in the current experiment for MMP-9 and GAPDH are as follows: MMP-9: sense, ATCAGCCGGGAACG TATCTG; antisense, GTTGTGGAAACTCACACG CC; GAPDH: sense, GTTACCAGGGCTGCCTTCTC; antisense, GTTACCAGGGCTGCCTTCTC.

\section{Histological studies}

After perfusion with PBS and ice-cold 4\% paraformaldehyde, brain tissues of four animals in each group were fixed in $4 \%$ paraformaldehyde and then dehydrated in graded ethanol until became transparent and then embedded in paraffin. The blocks were cut coronally into serial five $\mu \mathrm{m}$-thick sections. Selected sections from each block were treated with xylene and alcohol in different concentrations, rinsed in water and stained with $0.1 \%$ cresyl-violet acetate (Sigma-Aldrich, USA). The prepared slides were analyzed by the morphometric software (Optika Vision Pro, Italy). Surviving pyramidal neurons were quantified under 200× magnifications. Three fields of hippocampal CA1 regions were randomly selected. Intact neurons were counted, and the average number of neurons was calculated for analysis ${ }^{[30,31]}$.

\section{Statistical analysis}

Behavioral data were analyzed using repeated measures two-way ANOVA and one-way ANOVA. Comparison between the groups in the molecular and histological studies was made by one-way ANOVA. The post-hoc test was Tukey. Pearson correlation test was applied to investigate the association between gene expression and cognition. SPSS 16.0 software was used for statistical analysis. In all statistical analyses, $p$ values less than 0.05 was considered as significant. 


\section{RESULTS}

\section{Simvastatin showed no effect on locomotor activity in the open field test}

We performed open field test to evaluate locomotor activity after the recovery of stereotaxic surgery. The number of line crosses and rearing behavior during five min was recorded in each session. As presented in Table 1, there was no significant change between groups in the number of line crosses $[\mathrm{F}(3,28)=0.57]$. Moreover, no significant difference was detected in the number of rearing among different groups $[\mathrm{F}(3,28)=$ $0.96]$.

\section{Simvastatin improved learning and memory in the MWM test}

The MWM test was performed to assess the hippocampal-dependent learning process and spatial reference memory. Animals with cognitive impairment spent more time and traveled more distance to find the platform during the trials of MWM test. The total distance moved and escape latency are shown in Figure 1. We found a significant difference between the treated groups in escape latency $[\mathrm{F}(3,28)=202.97, p<$ 0.001 , Fig. 1A $]$ and distance traveled $[\mathrm{F}(3,28)=89.3$, $p<0.001$, Fig. 1B]. The Saline + DMSO group showed a progressive reduction in escape latency over successive trials, similar to the Saline + Sim group. The STZ + DMSO group compared with Saline + DMSO and Saline + Sim groups indicated significantly prolonged escape latency during trials on day three $(18.4 \pm 4.9$ or $19.9 \pm 2.3$ vs. $33.4 \pm 1.4 \mathrm{sec}, \mathrm{F}(3,28)=$ $4.52, p<0.05)$ and day four $(13.5 \pm 2.9$ or $14.0 \pm 0.7$ vs. $31.3 \pm 1.3 \mathrm{sec}, \mathrm{F}(3,28)=21.72, p<0.001)$. The mean escape latency to find the platform significantly improved in the STZ + Sim group compared with STZ + DMSO on both day three $(19.7 \pm 2.3$ vs. $33.4 \pm 1.4$ sec, $p<0.05)$ and day four $(15.0 \pm 1.6$ vs. $31.3 \pm 1.3$ sec, $p<0.001)$. The total distance traveled was significantly longer for the STZ + DMSO group compared with the Saline + DMSO and Saline + Sim on day three $(684.2 \pm 96$ or $537.6 \pm 94.0$ vs. $1097.2 \pm$ $127.2 \mathrm{~cm}, p<0.05)$ and day four $(476.0 \pm 92.1$ or 448.9 \pm 66.6 vs. $993.5 \pm 70.4 \mathrm{~cm}, p<0.001)$. The distance traveled significantly decreased in the STZ + Sim group in comparison to the STZ + DMSO group on both day three $(566.9 \pm 25.9$ vs. $1097.2 \pm 127.2 \mathrm{~cm}, p$ $<0.05)$ and day four $(551.6 \pm 84.6$ vs. $993.5 \pm 70.4 \mathrm{~cm}$, $p<0.001)$. Swimming velocity did not change significantly in different groups during four days of place navigation $(\mathrm{F}(3,28)=1.65, p>0.05$, Fig. $1 \mathrm{E})$.

In the probe test, our data showed significant difference between the groups in the percentage of time spent $[\mathrm{F}(3,28)=5.3, p<0.05$, Fig. $1 \mathrm{C}])$ and distance traveled $[\mathrm{F}(3,28)=6.9, p<0.001$, Fig. 1D] in the goal quadrant. The percentage of time exploring in the goal quadrant decreased in the STZ + DMSO compared with the Saline + DMSO and Saline + Sim groups $(16.7 \pm 2.8$ vs. $33.82 \pm 4.8$ and $33.80 \pm 1.6 p<0.05)$. The percentage of distance traveled in the goal quadrant in the Saline + DMSO group was similar to that of the Saline + Sim group, while it significantly decreased in the STZ + DMSO group in comparison with Saline + DMSO and Saline+Sim groups (37.1 \pm 1.8 and $44.7 \pm 8.3$ vs. $15.7 \pm 2.9, p<0.05$ ). However, the percentage time of exploration in the goal quadrant in the probe test significantly raised in the STZ $+\mathrm{Sim}$ group when compared to the STZ + DMSO group $(31.0 \pm 3.9$ vs. $16.7 \pm 2.8, p<0.05)$. The percentage of distance significantly elevated in the STZ + Sim group compared with the STZ + DMSO group $(38.2 \pm 3.1$ vs. $15.7 \pm 2.9, p<0.05)$.

\section{Simvastatin reduced MMP-9 gene expression in hippocampus}

Hippocampal MMP-9 gene expression level was evaluated using quantitative real-time PCR. The hippocampus tissues of four animals in each group were isolated for MMP-9 gene expression, and the PCR results of each animal were repeated for three consecutive times. As depicted in Figure 2, no significant difference was detected in MMP-9 expression between the Saline + DMSO and Saline + Sim groups. However, MMP-9 expression was upregulated in the STZ + DMSO group compared with Saline + DMSO and Saline + Sim groups $(1.41 \pm 0.02$ vs. $0.987 \pm 0.01$ and $0.980 \pm 0.02, p<0.05)$. Simvastatin administration significantly prevented the rise of MMP-9 expression in the STZ + Sim group relative to STZ + DMSO group $(1.05 \pm 0.05$ vs. $1.41 \pm$ $0.2, p<0.05)$.

\section{A negative correlation was found between hippocampal MMP-9 expression and cognitive indices}

As presented in Figure 3, Pearson correlation analysis demonstrated a significant negative relationship between MMP-9 gene expression in

Table 1. Data of general activity in different groups ( $\mathrm{n}=8$ in each group)

\begin{tabular}{lcc}
\hline \multirow{2}{*}{ Groups } & \multicolumn{2}{c}{ Open field test } \\
\cline { 2 - 3 } & $\begin{array}{c}\text { Number of } \\
\text { line crosses }\end{array}$ & $\begin{array}{c}\text { Frequency } \\
\text { of rearing }\end{array}$ \\
\hline Saline + DMSO & $46.7 \pm 2.57$ & $7.25 \pm 0.75$ \\
Saline + Sim & $46.75 \pm 4.51$ & $6.37 \pm 0.77$ \\
STZ + DMSO & $52.50 \pm 5.10$ & $8.25 \pm 1.10$ \\
STZ + Sim & $46.50 \pm 2.38$ & $6.62 \pm 0.59$ \\
\hline
\end{tabular}

Values are expressed as mean \pm SEM. No significant difference was seen between the groups. 

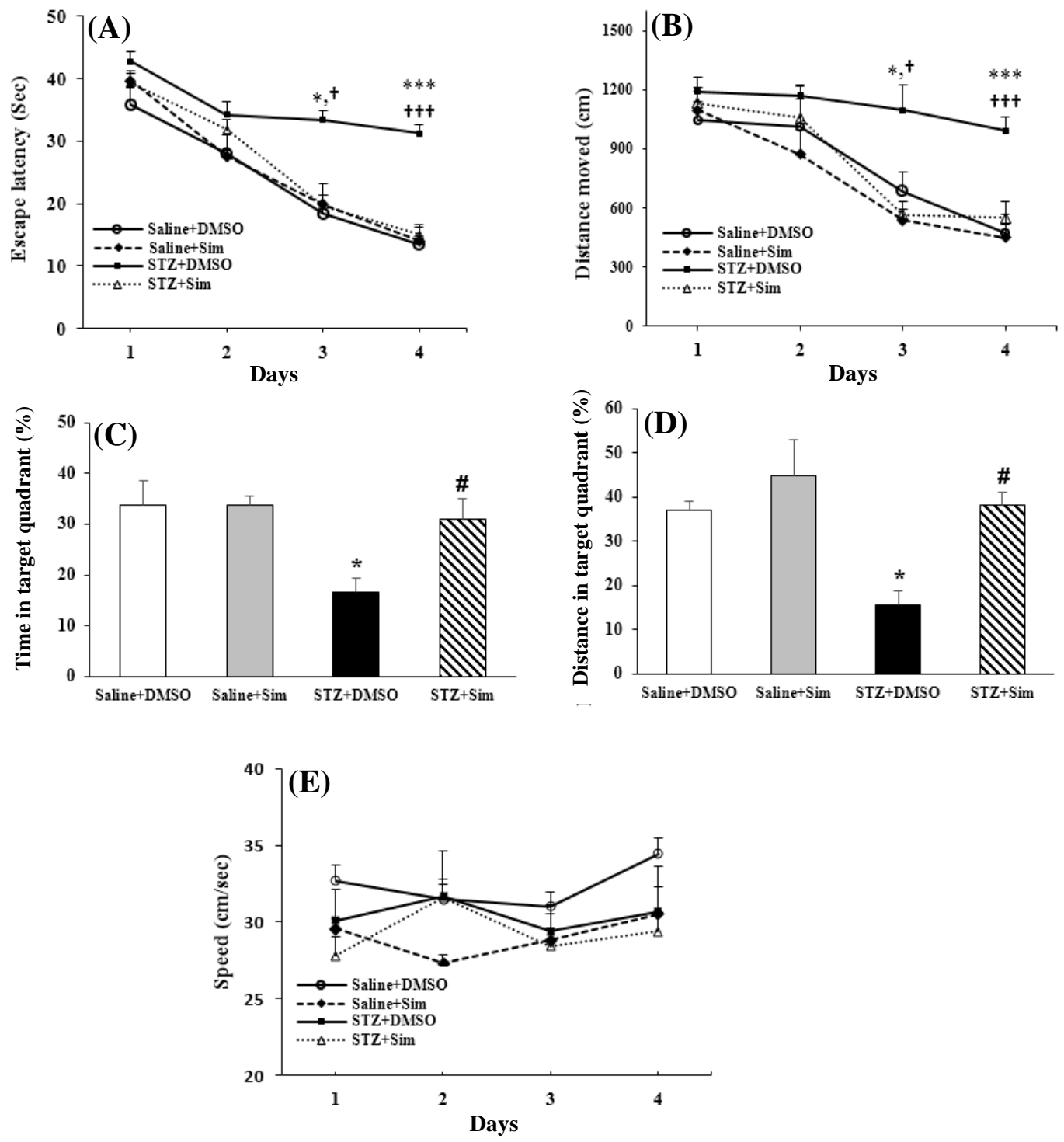

Fig. 1. Effects of simvastatin (Sim) administration on learning and memory function of rats in Morris water maze test $(\mathrm{n}=8$ in each group). During place navigation stages on days 17-20 following STZ injection, time taken for the rats to find the platform (a) and total distance traveled (b) in each trial were measured. Percentage of time (c) and distance (d) spent in the goal quadrant (platform area) during probe test is shown in the Figure. There were significant differences between STZ + Sim and STZ + DMSO in distance moved and escape latency during days three and four of place navigation stages. Swimming speed did not change significantly in different groups during four days of place navigation (e). Values are expressed as mean \pm SEM. ${ }^{*} p<0.05$ and $*^{* * *} p<0.001$ compared with Saline + DMSO group; ${ }^{\#} p<0.05$ compared with STZ + DMSO group; ${ }^{\dagger} p<0.05$ and ${ }^{\dagger \dagger \dagger} p<0.001$ compared with STZ + Sim group.

hippocampus and time spent $(\mathrm{r}=0.77$, Fig. $3 \mathrm{~A})$ and distance moved $(r=0.69$, Fig. 3B) in the goal quadrant (as cognitive indices) during probe test in the MWM test.

\section{Simvastatin improved the morphological features of hippocampal neurons}

Morphology and cellular arrangement of pyramidal neurons in hippocampus tissue were monitored by cresyl violet staining. Pyramidal neurons in the hippocampus tissues of the Saline + DMSO, Saline +
Sim, and Saline + Sim groups had normal morphology with densely packed arrangement. Damaged cells with shrunk cell bodies were noted in the hippocampus sections of the Saline + STZ group (Fig. 4A). There were significant changes in neuron count on different treatment samples between the groups $(\mathrm{F}(3,12)=$ $156.1, p<0.001)$. No significant difference was seen in hippocampal cell count in the Saline + DMSO and Saline + Sim groups. The neuron count in the STZ + DMSO group significantly reduced compared with the Saline + DMSO and Saline + Sim groups 


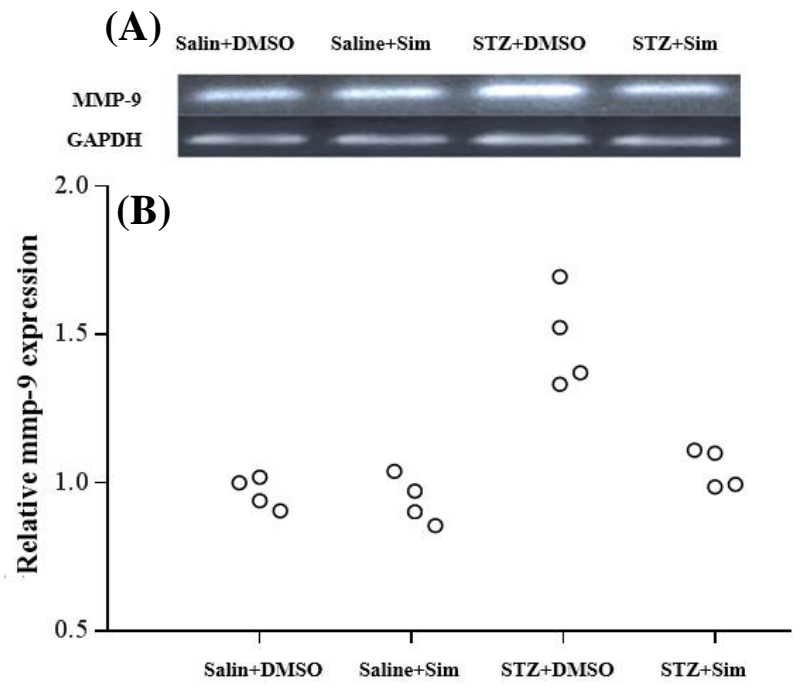

Fig 2. Relative MMP-9 expression in hippocampus tissue of different groups. (a) The gel electrophoresis of PCR products of MMP-9 and GAPDH in different groups; (b) the relative expression of MMP-9 gene in different groups. The hippocampus tissues were isolated from four animals in each group, and each hippocampus was assessed three replicate times. The circles show the data of each rat in a group.

(76.5 \pm 2.1 and $77.7 \pm 3.5$ vs $16.5 \pm 1.5, p<0.001)$. The neuron count in hippocampal sections increased in the STZ + Sim group relative to the STZ + DMSO group, as shown in Figure 4B $(75.5 \pm 1.8$ vs. $16.5 \pm$ $1.5, p<0.001)$. These data showed the neuroprotective effect of simvastatin on hippocampal pyramidal cells against neural damage induced by ICV-STZ injection.

\section{DISCUSSION}

In the present study, a significant cognitive decline, recognized by prolonged mean escape latency, was detected in the STZ group when compared to the control group. This observation is in line with previous findings regarding spatial memory decline in this model $^{[32,33]}$. The presence of shrunk cell bodies in hippocampus sections of the STZ group confirmed the induction of injury ${ }^{[34]}$. The present results showed that hippocampal MMP-9 up-regulated in the rat model of cognitive impairment induced by intracerebroventricular injection of STZ. Our results are in agreement with a previous finding that demonstrated MMP-9 involvement in the pathogenesis of brain injuries and cognitive disorders ${ }^{[35]}$. Elevated levels of MMP-9 were localized in the cytoplasm of neurons, neurofibrillary tangles, senile plaques, and vascular walls in postmortem sections of the parietal lobe and hippocampus from AD patients ${ }^{[5]}$. The greater proteolytic activity of MMP-9 in the postmortem frontal and parietal cortical tissues of AD patients has been observed ${ }^{[7,36]}$. MMP-9 is expressed in the various regions of brain including the hippocampus ${ }^{[37]}$ and localized and released from neurons, astrocytes, and microglia $^{[38]}$. MMP-9 overexpression activates cytokines and enhances neuro-inflammation and cell death ${ }^{[39]}$. Elevated level of MMP-9 has been reported in the transgenic mouse $\mathrm{AD}$ model ${ }^{[40]}$. Increased levels of MMP-9 expression have also been reported in the hippocampus and cerebral cortex of AD-affected patients ${ }^{[5,6]}$. Overexpression of MMP-9 is associated with disturbed hippocampal long-term potentiation $^{[41]}$. Therefore, the prolonged mean escape latency in the present study may in part be related to the overexpression of MMP-9 in the STZ group. One study, which performed a quantitative gel zymography, has found no change in plasma MMP-9 activity in AD patients ${ }^{[42]}$. This observation may partly be due to the discrepancies between the enzymatic activity and gene expression of MMP-9. Moreover, in this study, we found a negative correlation between MMP-9 expression and cognitive function in rats from all groups, which implies that MMP-9 level may contribute to cognition impairment in AD pathology. A number of studies have focused on MMP-9 gene expression in neurodegenerative disorders ${ }^{[8,20]}$.
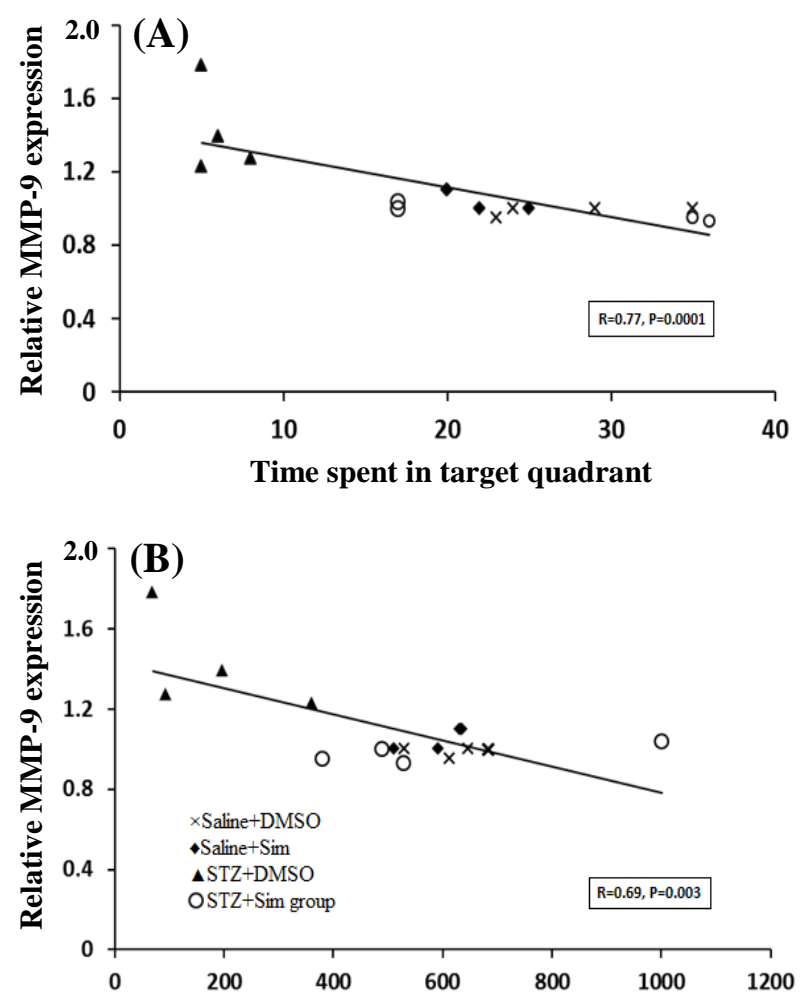

Distance traveled in target quadrant

Fig. 3. Correlation analysis between MMP-9 relative expression and time spent in the goal quadrant (a) and total distance traveled (b) in MWM, as indexes of cognitive function. 


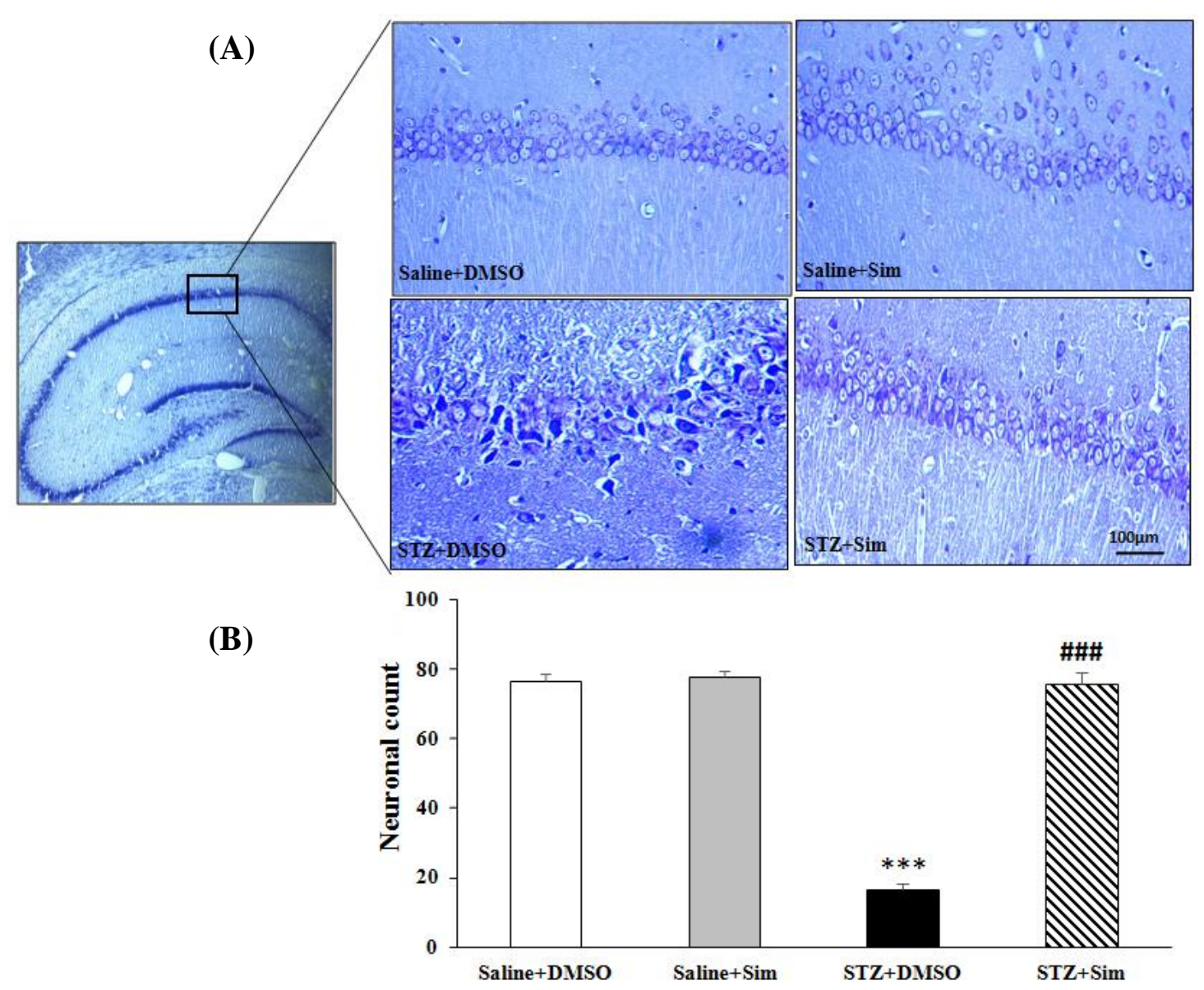

Fig. 4. Alteration of the CA1 hippocampal pyramidal cells morphology in different groups. Following the completion of the experiment, four hippocampal tissues from each group $(\mathrm{n}=4)$ were processed for cresyl violet staining. (a) Intact morphology and regular arrangement in Saline + DMSO, Saline + Sim, and STZ + Sim groups. In STZ + DMSO group, cell bodies were shrunken with unpacked arrangement in hippocampal tissue. (b) The neuronal count in the hippocampal CA1 regions in slide fields in different groups. Values are expressed as mean \pm SEM. ${ }^{* * *} p<0.001$ compared with Saline + DMSO; ${ }^{\# \# \# p} p 0.001$ compared with STZ + DMSO group.

Recently, statins have been noticed as effective neuroprotective molecules ${ }^{[43]}$. In our study, simvastatin prevented the rise of MMP-9 expression in the STZ group, decreased neural damage in hippocampal morphology and eventually improved spatial long term memory.

In the current work, administration of simvastatin for three weeks enhanced learning and memory in the STZ + Sim group. Many clinical studies have reported that statins have a significant effect on memory in patients $^{[44-46]}$, whereas some others have failed to demonstrate this beneficial effect ${ }^{[47,48]}$. This controversy in clinical reports may be, to some extent, due to the variations in the study design, dose and duration of statin administration, and methods of cognition examination ${ }^{[49]}$.

As presented in the current study, simvastatin improved neural density in hippocampal tissue in the
STZ + Sim group. Another study has shown suppressed neural death in the hippocampus by simvastatin $^{[49]}$. Simvastatin modulates MMP-9 expression and reduces neuro-inflammation and apoptosis $^{[15,50]}$. It has been reported that simvastatin hinders oxidative stress through increasing the expression of hippocampal antioxidant enzymes ${ }^{[51]}$. It has also been reported that simvastatin improves memory impairment after cerebrovascular damage ${ }^{[52,53]}$, prevents oxidative damages, and enhances long-term potentiation ${ }^{[25,54]}$. These protective actions may explain the enhancement in hippocampal cell count and neuronal density in the ICV-STZ injected rats.

Oral administration of simvastatin, $5 \mathrm{mg} / \mathrm{kg}$ body weight for three weeks after STZ infusion, prevented the up-regulation of MMP-9 gene expression, reduced neuronal damage in hippocampal tissue and 
subsequently improved cognitive impairment. We also observed negative correlation between MMP-9 gene expression and spatial memory capability. Our findings suggest that the modulation of MMP-9 gene expression may be a neuroprotective scheme of simvastatin in battle with the cognitive decline in AD.

\section{ACKNOWLEDGMENTS}

This study was financially supported by a grant (No. 93-01-87-24462) from Tehran University of Medical Sciences, Tehran, Iran.

CONFLICT OF INTEREST. None declared.

\section{REFERENCES}

1. De Luca C, Papa M. Matrix metalloproteinases, neural extracellular matrix, and central nervous system pathology. Progress in molecular biology and translational science 2017; 148: 167-202.

2. Demir R, Ulvi H, Özel L, Özdemir G, Güzelcik M, Aygül R. Relationship between plasma metalloproteinase-9 levels and volume and severity of infarct in patients with acute ischemic stroke. Acta neurologica belgica 2012; 112(4): 351-356.

3. Lorenzl S, Büerger K, Hampel H, Beal MF. Profiles of matrix metalloproteinases and their inhibitors in plasma of patients with dementia. International psychogeriatrics 2008; 20(1): 67-76.

4. Suehiro E, Fujisawa $H$, Akimura $T$, Ishihara $H$, Kajiwara K, Kato S, Fujii M, Yamashita S, Maekawa T, Suzuki M. Increased matrix metalloproteinase-9 in blood in association with activation of interleukin-6 after traumatic brain injury: influence of hypothermic therapy. Journal of neurotrauma 2004; 21(12): 17061711.

5. Asahina M, Yoshiyama Y, Hattori T. Expression of matrix metalloproteinase-9 and urinary-type plasminogen activator in Alzheimer's disease brain. Clinical neuropathology 2001; 20(2): 60-63.

6. Backstrom JR, Miller CA, Tökés ZA. Characterization of neutral proteinases from Alzheimer-affected and control brain specimens: identification of calciumdependent metalloproteinases from the hippocampus. Journal of neurochemistry 1992; 58(3): 983-992.

7. Bruno MA, Mufson EJ, Wuu J, Cuello AC. Increased matrix metalloproteinase 9 activity in mild cognitive impairment. Journal of neuropathology and experimental neurology 2009; 68(12): 1309-1318.

8. Mizoguchi H, Takuma K, Fukuzaki E, Ibi D, Someya E, Akazawa KH, Alkam T, Tsunekawa H, Mouri A, Noda Y, Nabeshima T, Yamada K. Matrix metalloprotease-9 inhibition improves amyloid $\beta$-mediated cognitive impairment and neurotoxicity in mice. The journal of pharmacology and experimental therapeutics 2009;
331(1): 14-22.

9. Gu Z, Cui J, Brown S, Fridman R, Mobashery S, Strongin AY, Lipton SA. A highly specific inhibitor of matrix metalloproteinase-9 rescues laminin from proteolysis and neurons from apoptosis in transient focal cerebral ischemia. Journal of neuroscience 2005; 25(27): 6401-6408.

10. Haag MD, Hofman A, Koudstaal PJ, Stricker BH, Breteler MM. Statins are associated with a reduced risk of Alzheimer disease regardless of lipophilicity. The Rotterdam Study. Journal of neurology, neurosurgery and psychiatry 2009; 80(1): 13-17.

11. Saheki A, Terasaki T, Tamai I, Tsuji A. In vivo and in vitro blood-brain barrier transport of 3-hydroxy-3methylglutaryl coenzyme A (HMG-CoA) reductase inhibitors. Pharmaceutical research 1994; 11(2): 305311 .

12. Saeedi Saravi SS, Saeedi Saravi SS, Arefidoust A, Dehpour AR. The beneficial effects of HMG-CoA reductase inhibitors in the processes of neurodegeneration. Metabolic brain disease 2017; 32(4): 1-17.

13. Li B, Mahmood A, Lu D, Wu H, Xiong Y, Qu C, Chopp M. Simvastatin attenuates microglial cells and astrocyte activation and decreases interleukin-1beta level after traumatic brain injury. Neurosurgery 2009; 65(1): 179186.

14. McFarland A, Davey A, Anoopkumar-Dukie S. Statins reduce lipopolysaccharide-induced cytokine and inflammatory mediator release in an in vitro model of microglial-like cells. Mediators of inflammation 2017; 2017: 2582745.

15. Huang W, Li Z, Zhao L, Zhao W. Simvastatin ameliorate memory deficits and inflammation in clinical and mouse model of Alzheimer's disease via modulating the expression of miR-106b. Biomedicine and pharmacotherapy 2017; 92: 46-57.

16. Griffin JM, Kho D, Graham ES, Nicholson LF, O'Carroll SJ. Statins inhibit fibrillary $\beta$-amyloid induced inflammation in a model of the human blood brain barrier. PLoS one 2016; 11(6): e0157483.

17. Chang TI, Kang HY, Kim KS, Lee SH, Nam BY, Paeng J, Kim S, Park JT, Yoo TH, Kang SW, Han SH. The effect of statin on epithelial-mesenchymal transition in peritoneal mesothelial cells. PLoS one 2014; 9(10): e109628.

18. Chen YJ, Chang LS. Simvastatin induces NFkB/p65 down-regulation and JNK1/c-Jun/ATF-2 activation, leading to matrix metalloproteinase-9 (MMP-9) but not MMP-2 down-regulation in human leukemia cells. Biochemical pharmacology 2014; 92(4): 530-543.

19. Andrade VL, do Valle IB, Sandrim VC. Simvastatin therapy decreases MMP-9 levels in obese women. The Journal of clinical pharmacology 2013; 53(10): 10721077.

20. Kaplan A, Spiller KJ, Towne C, Kanning KC, Choe GT, Geber A, Akay T, Aebischer P, Henderson CE. Neuronal matrix metalloproteinase- 9 is a determinant of selective neurodegeneration. Neuron 2014; 81(2): 333348. 
21. Yan J, Xu Y, Zhu C, Zhang L, Wu A, Yang Y, Xiong Z, Deng C, Huang XF, Yenari MA, Yang YG, Ying W, Wang Q. Simvastatin prevents dopaminergic neurodegeneration in experimental parkinsonian models: the association with anti-inflammatory responses. PLoS one 2011; 6(6): e20945.

22. Labak M, Foniok T, Kirk D, Rushforth D, Tomanek B, Jasiński A, Grieb P. Metabolic changes in rat brain following intracerebroventricular injections of streptozotocin: a model of sporadic Alzheimer's disease. Acta neurochirurgica supplement 2010; 106: 177-181.

23. Salkovic-Petrisic M, Knezovic A, Hoyer S, Riederer P. What have we learned from the streptozotocin-induced animal model of sporadic Alzheimer's disease, about the therapeutic strategies in Alzheimer's research. Journal of neural transmission (Vienna) 2013; 120(1): 233-252.

24. Grieb P. Intracerebroventricular streptozotocin injections as a model of Alzheimer's disease: in search of a relevant mechanism. Molecular neurobiology 2016; 53(3): 1741-1752.

25. Chen Y, Liang Z, Blanchard J, Dai CL, Sun S, Lee MH, Grundke-Iqbal I, Iqbal K, Liu F, Gong CX. A nontransgenic mouse model (icv-STZ mouse) of Alzheimer's disease: similarities to and differences from the transgenic model $(3 \times \mathrm{Tg}-\mathrm{AD}$ mouse). Molecular neurobiology 2013; 47(2): 711-725.

26. Salkovic-Petrisic M, Hoyer S. Central insulin resistance as a trigger for sporadic Alzheimer-like pathology: an experimental approach. Journal of neural transmission 2007; 72: 217-233.

27. Nazari A, Zahmatkesh M, Mortaz E, Hosseinzadeh S. Effect of methamphetamine exposure on the plasma levels of endothelial-derived microparticles. Drug and alcohol dependence 2018; 186: 219-225.

28. Morris R. Developments of a water-maze procedure for studying spatial learning in the rat. Journal of neuroscience methods 1984; 11(1): 47-60.

29. Rao X, Huang X, Zhou Z, Lin X. An improvement of the $2^{\wedge}$ (-delta delta CT) method for quantitative real-time polymerase chain reaction data analysis. Biostatistics, bioinformatics and biomathematics 2013; 3(3): 71-85.

30. Huang Y, Lai H, Xu H, Wu W, Lai X, Ho G, Ma L, Chen Y. Impact of perinatal systemic hypoxic-ischemic injury on the brain of male offspring rats: An improved model of neonatal hypoxic-ischemic encephalopathy in early preterm newborns. PloS one 2013; 8(12): e82502

31. Zhang Z, Sun T, Niu JG, He ZQ, Liu Y, Wang F. Amentoflavone protects hippocampal neurons: antiinflammatory, antioxidative, and antiapoptotic effects. Neural regeneration research 2015; 10(7): 1125-1133.

32. Hosseinzadeh S, Zahmatkesh M, Heidari M, Hassanzadeh GR, Karimian M, Sarrafnejad A, Zarrindast MR. Hippocampal DHCR24 down regulation in a rat model of streptozotocin-induced cognitive decline. Neuroscience letters 2015; 587: 107-112.

33. Adel Ghahraman M, Zahmatkesh M, Pourbakht A, Seifi B, Jalaie S, Adeli S, Niknami Z. Noisy galvanic vestibular stimulation enhances spatial memory in cognitive impairment-induced by intracerebroventricular-streptozotocin administration. Physiology and behavior 2016; 157: 217-224.

34. Esmaeilzade B, Nobakht M, Joghataei MT, Rahbar Roshandel N, Rasouli H, Samadi Kuchaksaraei A, Hosseini SM, Najafzade N, Asalgoo S, Hejazian LB, Moghani Ghoroghi F. Delivery of epidermal neural crest stem cells (EPI-NCSC) to hippocamp in Alzheimer's disease rat model. Iranian biomedical journal 2012; 16(1): 1-9.

35. Van Der Kooij MA, Fantin M, Rejmak E, Grosse J, Zanoletti O, Fournier C, Ganguly K, Kalita K, Kaczmarek L, Sandi C. Role for MMP-9 in stressinduced downregulation of nectin-3 in hippocampal CA1 and associated behavioural alterations. Nature communications 2014; 5: Article number 4995.

36. Halliday MR, Rege SV, Ma Q, Zhao Z, Miller CA, Winkler EA, Zlokovic BV. Accelerated pericyte degeneration and blood-brain barrier breakdown in apolipoprotein E4 carriers with Alzheimer's disease. Journal of cerebral blood flow and metabolism 2016; 36(1): 216-227.

37. Szklarczyk A, Lapinska J, Rylski M, McKay RD, Kaczmarek L. Matrix metalloproteinase-9 undergoes expression and activation during dendritic remodeling in adult hippocampus. Journal of neuroscience 2002; 22(3): 920-930.

38. Gottschall PE, Deb S. Regulation of matrix metalloproteinase expression in astrocytes, microglia and neurons. Neuroimmunomodulation 1996; 3(2-3): 69-75.

39. Leonardo CC, Hall AA, Collier LA, Gottschall PE, Pennypacker KR. Inhibition of gelatinase activity reduces neural injury in an ex vivo model of hypoxiaischemia. Neuroscience 2009; 160(4): 755-766.

40. Kurata T, Kawai H, Miyazaki K, Kozuki M, Morimoto N, Ohta Y, Ikeda Y, Abe K. Statins have therapeutic potential for the treatment of Alzheimer's disease, likely via protection of the neurovascular unit in the AD brain. Journal of the neurological sciences 2012; 322(1): 5963.

41. Wiera G, Szczot M, Wojtowicz T, Lebida K, Koza P, Mozrzymas J. Impact of matrix metalloproteinase-9 overexpression on synaptic excitatory transmission and its plasticity in rat CA3-CA1 hippocampal pathway. Canadian journal of physiology and pharmacology 2015; 66(2): 309-315.

42. Martín-Aragón S, Bermejo-Bescós P, Benedí J, Felici E, Gil P, Ribera JM, Villar ÁM. Metalloproteinase's activity and oxidative stress in mild cognitive impairment and Alzheimer's disease. Neurochemical research 2009; 34(2): 373-378.

43. Carroll CB, Wyse RK. Simvastatin as a potential disease-modifying therapy for patients with Parkinson's disease: rationale for clinical trial, and current progress. Journal of Parkinson's disease 2017; 7(4): 545-568.

44. Geifman N, Brinton RD, Kennedy RE, Schneider LS, Butte AJ. Evidence for benefit of statins to modify cognitive decline and risk in Alzheimer's disease. Alzheimer's research and therapy 2017; 9: 10.

45. Heymann AD, Ravona-Springer R, Moshier EL, Godbold J, Beeri MS. Statin use is associated with 
better cognitive function in elderly with type 2 diabetes. Journal of Alzheimer's disease 2015; 47(1): 55-59.

46. Swiger KJ, Manalac RJ, Blumenthal RS, Blaha MJ, Martin SS. Statins and cognition: a systematic review and meta-analysis of short-and long-term cognitive effects. Mayo clinic proceedings 2013; 88(11): 12131221.

47. Moryś JM, Kozera GM, Neubauer-Geryk J, Kruszewski P, Wolnik B, Nyka WM, Bieniaszewski L. Statin use and cognitive impairment in patients with type 1 diabetes: An observational study. Clinical neuropharmacology 2016; 39(4): 182-187.

48. Samaras K, Brodaty H, Sachdev PS. Does statin use cause memory decline in the elderly? Trends in cardiovascular medicine 2016; 26(6): 550-565.

49. Fang SC, Xie H, Chen F, Hu M, Long Y, Sun HB, Kong LY, Hong H, Tang SS. Simvastatin ameliorates memory impairment and neurotoxicity in streptozotocin-induced diabetic mice. Neuroscience 2017; 355: 200-211.

50. JCE MJ, Macedo CG, Abdalla HB, Saba AK, Teixeira LN, Mouchrek AQES, Napimoga MH, ClementeNapimoga JT, Borges AH, Tonetto MR, Pinto SCS, Bandeca MC, Martinez EF. Simvastatin modulates gingival cytokine and MMP production in a rat model of ligature-induced periodontitis. Clinical, cosmetic and investigational dentistry 2017; 9: 33-38.

51. Catalão CHR, Santos-Júnior NN, da Costa LHA, Souza AO, Alberici LC, Rocha MJA. Brain oxidative stress during experimental sepsis is attenuated by simvastatin administration. Molecular neurobiology 2017; 54(9): 7008-7018

52. Tong X-K, Lecrux C, Rosa-Neto P, Hamel E. Agedependent rescue by simvastatin of Alzheimer's disease cerebrovascular and memory deficits. Journal of neuroscience 2012; 32(14): 4705-4715.

53. Roy A, Jana M, Kundu M, Corbett GT, Rangaswamy SB, Mishra RK, Luan C-H, Gonzalez FJ, Pahan K. HMG-CoA reductase inhibitors bind to PPAR $\alpha$ to upregulate neurotrophin expression in the brain and improve memory in mice. Cell metabolism 2015; 22(2): 253-265.

54. Parent MA, Hottman DA, Cheng S, Zhang W, McMahon LL, Yuan LL, Li L. Simvastatin treatment enhances NMDAR-mediated synaptic transmission by upregulating the surface distribution of the GluN2B subunit. Cellular and molecular neurobiology 2014; 34(5): 693-705. 